University of South Carolina

Scholar Commons

$12-15-2005$

\title{
Laser-Controlled Local Magnetic Field with Semiconductor Quantum Rings
}

Yuriy V. Pershin Dr

University of South Carolina - Columbia, pershin@physics.sc.edu

Carlo Piermarocchi

Follow this and additional works at: https://scholarcommons.sc.edu/phys_facpub

Part of the Physics Commons

Publication Info

Published in Physical Review B, ed. Gene D. Sprouse, Volume 72, Issue 24, 2005, pages

245331-1-245331-5.

Pershin, Y. V., \& Piermarocchi, C. (2005). Laser-controlled local magnetic field with semiconductor quantum rings. Physical Review B, 72(24), 245331-1 - 245331-5. DOI: 10.1103/PhysRevB.72.245331 (c) Physical Review B, 2005, American Physical Society

This Article is brought to you by the Physics and Astronomy, Department of at Scholar Commons. It has been accepted for inclusion in Faculty Publications by an authorized administrator of Scholar Commons. For more information, please contact digres@mailbox.sc.edu. 


\title{
Laser-controlled local magnetic field with semiconductor quantum rings
}

\author{
Yuriy V. Pershin* and Carlo Piermarocchi \\ Department of Physics and Astronomy, Michigan State University, East Lansing, Michigan 48824, USA
}

(Received 12 October 2005; published 21 December 2005)

\begin{abstract}
We analyze theoretically the dynamics of $N$ electrons localized in a narrow semiconductor quantum ring under a train of phase-locked infrared laser pulses. The pulse sequence is designed to control the total angular momentum of the electrons. The quantum ring can be put in states characterized by strong currents. The local magnetic field created by these currents can be used for a selective quantum control of single spins in semiconductor systems. The current generation in quantum rings with finite width is also studied.
\end{abstract}

DOI: 10.1103/PhysRevB.72.245331

PACS number(s): 73.23.Ra, 78.67.-n

\section{INTRODUCTION}

The quantum control by trains of phase-locked laser pulses is a powerful and intuitive technique that has been applied to many atomic and molecular systems. It has been successfully employed in controlling the angular momentum of Rydberg electrons and molecular wavepackets, ${ }^{1}$ and it was the key technique in the creation of Schrödinger cat's states in Rydberg atoms. ${ }^{2}$ Semiconductor quantum dots and rings are artificial atoms with energy levels that can be engineered, and the realization of optical control in these systems is particularly appealing for quantum device applications.

In this paper we propose a scheme involving phase-locked infrared pulses to control the total angular momentum of $N$ electrons in a quantum ring. This implies that a strong current can be generated in the ring. The latter can be exploited to generate and control a local magnetic field in spin-based quantum computers. ${ }^{3,4}$ A possible application of the radiation-induced currents to single spin control is shown in Fig. 1(a). A spin, provided e.g., by a magnetic impurity, is embedded at the center or on top of a narrow quantum ring and can be locally controlled by the magnetic field due to the current in the ring. A scheme that uses arrays of parallel wires to create localized magnetic fields has been recently proposed..$^{5}$ The significant advantage of our laser-controlled approach is that it does not require external leads: the magnetic field is controlled by laser pulses. The peculiar orbital properties of the many-body eigenstates in a quantum ring and Pauli blocking effects make this scheme robust against the relaxation of the current by phonon and photon emission.

Small semiconductor nanorings can be made by the same self-assembly methods used for the fabrication of quantum dots. Persistent currents due to the Aharonov-Bohm flux have been observed in these quantum structures in the presence of an external magnetic flux along the ring axis. ${ }^{6}$ Currently, there is great theoretical interest in persistent currents. ${ }^{7-14}$ In particular, it is believed that they can be generated, besides using an external magnetic flux, also by the interplay of spin-orbit interaction and hyperfine coupling. ${ }^{7,8}$ We propose here that circularly polarized radiation can create currents in a quantum ring without an external magnetic flux. The circular polarization of the light propagating along the axis of the ring breaks the clockwise-anticlockwise symmetry of the many-electron wave function. In the case of a cw excitation these currents are persistent currents: they are as- sociated to the ground state of the ring dressed by the external laser field. Pulsed lasers can however produce stronger currents in a shorter time, which is more interesting for our purpose. We will discuss the $\mathrm{cw}$ case elsewhere. Notice that linearly polarized excitation will not induce a current but can affect the current induced by an external magnetic flux. ${ }^{10}$

Our paper is organized as follows. In Sec. II we consider the mechanism of current generation in narrow quantum rings. Using the density matrix formalism we study the population evolution in a quantum ring containing three electrons. The current generation in two-dimensional quantum rings is studied in Sec. III. The results of our investigation are summarized and discussed in Sec. IV.

\section{NARROW RINGS}

We start from $\Psi_{0}\left(\varphi_{1}, \ldots, \varphi_{N}\right)$, the exact $N$-electron ground state of a narrow ring Hamiltonian

$$
H_{R}=-\frac{\hbar^{2}}{2 m^{*} a^{2}} \sum_{i=1}^{N} \frac{\partial^{2}}{\partial \varphi_{i}^{2}}+U\left(\varphi_{1}, \ldots, \varphi_{N}\right)
$$

$a$ is the ring radius, $m^{*}$ is the electron effective mass, and $\varphi_{i}$ are the axial coordinates of the electrons. $U\left(\left\{\varphi_{i}\right\}\right)$ is the electron-electron Coulomb interaction. The operator $\hat{L}_{z}$ $=-i \sum_{i=1}^{N} \partial / \partial \varphi_{i}$ commutes with the $H_{R}$ and $\hat{L}_{z} \Psi_{0}=0$. We want to design a multipulse control Hamiltonian of the form

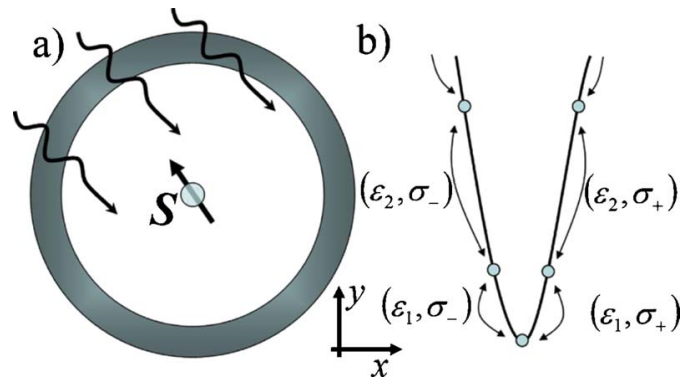

FIG. 1. (Color online) (a) Scheme of the system: circularly polarized light control of a spin by exciting currents in a quantum ring. (b) Scheme of the energy levels and selection rules for $N=1$. 


$$
H_{C}(t)=-\sum_{j} \mathbf{d} \cdot \mathbf{E}_{j}\left(t-\tau_{j}\right),
$$

where $\mathbf{d}$ is the total dipole moment of the $N$ electrons and $\mathbf{E}_{j}$ is the electric field of the $j$ th pulse. The target states in our scheme are written as

$$
\Psi_{l}=e^{i l\left(\varphi_{1}+\cdots+\varphi_{N}\right)} \Psi_{0},
$$

with $l$ integer, and are exact eigenstates of the Hamiltonian in Eq. (1) with energy $E_{l}=E_{0}+\hbar^{2} N l^{2} /\left(2 m^{*} a^{2}\right)$ and with $\hat{L}_{z} \Psi_{l}$ $=N l \Psi_{l}$. These states are called compact states and they can be seen as rigid rotational modes of the many-body electron system as a whole. The wave functions $\Psi_{0}$ and $\Psi_{l}$ are linked by a rotating frame transformation and their Coulomb correlation properties are identical. Every time a $\sigma_{+}$polarized photon is absorbed or emitted the total $L_{z}$ increases or decreases by one, while the opposite is valid for $\sigma_{-}$.

Let us consider first the dynamics of a single electron confined in the ring neglecting relaxation processes. The energy, wave function, and current of an electron in the $n$th level are given by

$$
\varepsilon_{n}=\frac{\hbar^{2} n^{2}}{2 m^{*} a^{2}}, \quad \psi_{n}=\frac{1}{\sqrt{2 \pi a}} e^{i n \varphi}, \quad i_{n}=\frac{e \hbar}{2 \pi a^{2} m^{*}} n .
$$

The time-dependent electric field $\mathbf{E}_{j}(t)$ generates transitions between the levels $\varepsilon_{n}$. Assuming square pulses, we write the electric field associated with the $j$ th pulse as $\mathbf{E}_{j}(t)$ $=E_{0 j} \cos \left(\omega_{j} t\right) \hat{x}+E_{1 j} \sin \left(\omega_{j} t\right) \hat{y}$ which accounts for arbitrary polarization. $E_{1}=E_{0}$ corresponds to $\sigma_{+}$polarization and $E_{1}$ $=-E_{0}$ to $\sigma_{-}$polarization. Matrix elements for dipole transitions are nonzero only between nearest single-particle states $\left\langle n\left|-d E_{ \pm}\right| n+1\right\rangle=e a E_{0} e^{ \pm i \omega t} / 2$, where \pm denotes $\sigma_{ \pm}$radiation. By writing the electron wave function in the form $\Psi$ $=\Sigma c_{n}(t) \psi_{n}$, where $c_{n}^{2}(t)$ gives the probability to find the electron in the state $n$, we get, for $\sigma_{+}$polarization, the equation of motion

$$
i \hbar \dot{c}_{n}=\varepsilon_{n} c_{n}+\frac{\alpha}{2} e^{i \omega t} c_{n+1}+\frac{\alpha}{2} e^{-i \omega t} c_{n-1},
$$

where $\alpha=e a E_{0}$ is the Rabi energy. The unitary transformation $c_{n}=e^{-i n \omega t} b_{n}$ allows us to eliminate the exponential factors in Eq. (5)

$$
i \hbar \dot{b}_{n}=\left(\varepsilon_{n}-n \hbar \omega\right) b_{n}+\frac{\alpha}{2} b_{n+1}+\frac{\alpha}{2} b_{n-1} .
$$

From Eq. (6) it follows that resonant transitions between two levels occur when $\widetilde{\varepsilon}_{n}=\widetilde{\varepsilon}_{n+1}$, where $\widetilde{\varepsilon}_{n}=\varepsilon_{n}-n \hbar \omega$. Notice that $\widetilde{\varepsilon}_{n+1}-\widetilde{\varepsilon}_{n}<0$ for any $n<0$ and $\omega>0$, meaning that $\sigma_{+}$radiation can only excite resonant transitions between levels with $n \geqslant 0$. For $\sigma_{-}$polarization, the dynamics of $b_{n}$ is described by Eq. (6) with $\omega$ replaced by $-\omega$. It can be shown that $\sigma_{-}$ radiation can only excite resonant transitions between levels with $n \leqslant 0$. The selection rules for single electron transitions are shown in Fig. 1(b).

Consider now $\mathrm{N}$-electron states of spinless and noninteracting electrons. Starting from a ground state, like the one in Fig. 2(a) for $N=3$, and using a sequence of pulses, our goal is

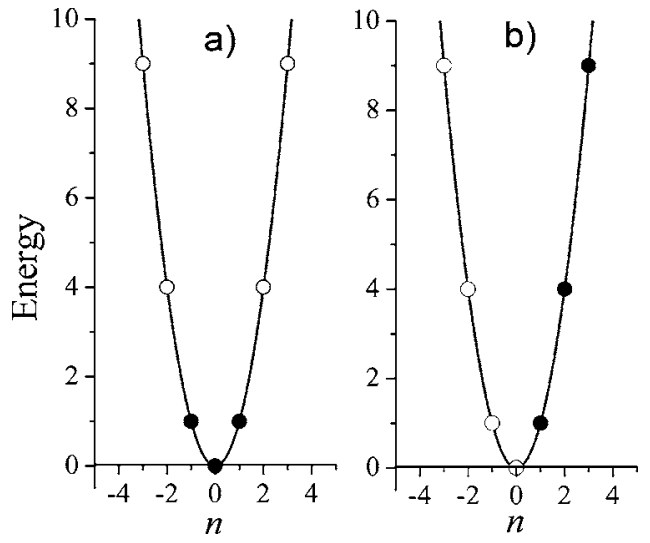

FIG. 2. Energy level population scheme: the ground state (a) and a compact excited state (b). The equilibrium ground state (a) is characterized by zero current. The current in the excited state (b) is strong since all electrons occupy the states with the same sign of the angular momentum.

to put the electrons in a compact state that is characterized by a strong current like, e.g., the one in Fig. 2(b). If in the ground state the levels $n=-N_{1}, \ldots, N_{1}$ are populated, in the final compact state the levels $n=\left(-N_{1}+p\right), \ldots,\left(N_{1}+p\right)$ are populated, i.e., $p$ electrons are moved from the states with negative angular momentum to the states with positive angular momentum. This is accomplished using a sequence of $\pi$ pulses with frequency and polarization $\left(\omega_{N+1}, \sigma_{+}\right)$, $\left(\omega_{N}, \sigma_{+}\right), \ldots,\left(\omega_{1}, \sigma_{+}\right),\left(\omega_{1}, \sigma_{-}\right), \ldots,\left(\omega_{N}, \sigma_{-}\right),\left(\omega_{N+2}, \sigma_{+}\right), \ldots$, where $\hbar \omega_{n}=\varepsilon_{n}-\varepsilon_{n-1}$. Notice that (i) each pulse increases $L_{z}$ by one by stimulated absorption of a $\sigma_{+}$or stimulated emission of a $\sigma_{-}$photon, and (ii) at each step in the sequence the energy of the optical transition is the smallest allowed by the many particle energy configuration on condition that $L_{z}$ increases by one. This implies that in our path toward the target compact states we follow the lowest energy states with fixed total $L_{z}$ (yrast line of excitations). From an operative standpoint, the polarization and the energy of the $j$ pulse in the sequence can be directly determined by spectroscopic measurements on the system. The energy of the photon to be used corresponds to $\min \left[\omega_{<}^{+}, \omega_{<}^{-}\right]$where $\omega_{<}^{+}$is the lowest $\sigma_{+}$-polarized peak in absorption, and $\omega_{<}^{-}$is the lowest $\sigma_{-}$-polarized peak in emission and/or gain. In the ideal case the final current is

$$
I=\sum_{n=-N_{1}+p}^{N_{1}+p} j_{n}=p\left(2 N_{1}+1\right) i_{1},
$$

which is $\simeq 2 p$ times stronger than the typical amplitude of the persistent current oscillations induced by the AharonovBohm flux. We would like to notice that the case $N_{1}=0$ in Eq. (7) corresponds to $N=1$ and $I=i_{p}$ in this situation.

The general idea of optical transitions with the smallest exchange of energy can be generalized to include the spin of the electrons. Notice that in the absence of spin-orbit coupling, the total spin of the electrons is conserved in the transitions. We have found that if a spin-orbit coupling of the Rashba form is present, this control scheme can create spinpolarized currents in the ring. ${ }^{15}$ Many-body calculations 


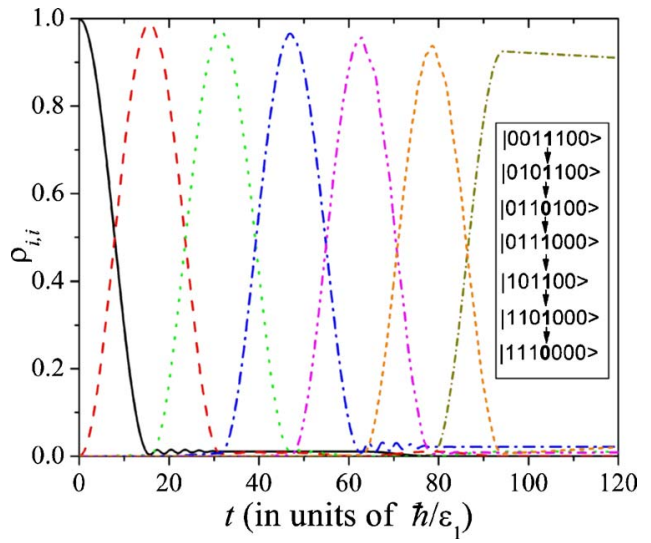

FIG. 3. (Color online) Evolution of the population of threeelectron levels induced by the pulse sequence. Inset: ideal sequence of transitions. The Rabi energy in the calculation is $\alpha=0.2 \varepsilon_{1}$ and the relaxation rate is $\gamma=0.02 \varepsilon_{1}$.

which were done using the configuration interaction method $^{16}$ show that the yrast line of excitations is always well separated from high excited states in small rings. Therefore in that case our general prescription for the pulse sequence to target compact states will not be affected by the presence of Coulomb interaction. The total exchange of energy at the end of the pulse sequence does not depend on the Coulomb interaction. The electromagnetic field provides only the kinetic energy necessary to a rigid rotation of all the electrons, since the potential energy of all compact states is the same as the one in the ground state.

Off-resonant population transfer and relaxation will affect our control scheme. We give a full description of the electron dynamics in the ring using a density matrix formalism. We include all nonresonant transitions without rotation wave approximation in the simulation. The equation of motion of the density matrix is given by $\dot{\rho}=-i / \hbar[H, \rho]+D\{\rho\}$ where the relaxation superoperator $D\{\rho\}$ has the form $D\{\rho\}$ $=\Sigma_{\xi}\left(\mathbf{L}_{\xi} \rho \mathbf{L}_{\xi}^{\dagger}-\frac{1}{2} \mathbf{L}_{\xi}^{\dagger} \mathbf{L}_{\xi} \rho-\frac{1}{2} \rho \mathbf{L}_{\xi}^{\dagger} \mathbf{L}_{\xi}\right)$, with Lindblad operators $\mathbf{L}_{\xi}$ $=\gamma_{\xi}|m\rangle\left\langle\left. n\right|^{\xi}\right.$ describing decoherence and relaxation processes. ${ }^{17}$

In order to demonstrate our approach, we solve the Liouville equation numerically assuming that three electrons are in the ring. Limiting our consideration to seven possible single-particle states (shown in Fig. 2), the ground state of the system can be represented as $|0011100\rangle$, where the 1 indicates an occupied level and 0 an empty level. The $n=0$ level is identified by a boldface index. There are 35 possible states that can be obtained with different occupations of seven levels by three electrons. We start from the configuration where only the ground state is populated, assuming that $\varepsilon_{2}-\varepsilon_{1} \gg k_{B} T$. The optical control is realized with pulses of rectangular shape. We select the pulse duration $T_{p}$ to obtain $\pi$ pulses as $\alpha T_{p} / \hbar=\pi$.

Figure 3 shows the result of our calculations. The applied pulse sequence generates almost perfect transitions between the states. The diagonal matrix elements in Fig. 3 correspond to the states indicated in the inset. We note that off-resonance transitions and relaxation result in the decrease of the maximum of population inversion at long times. The small fast

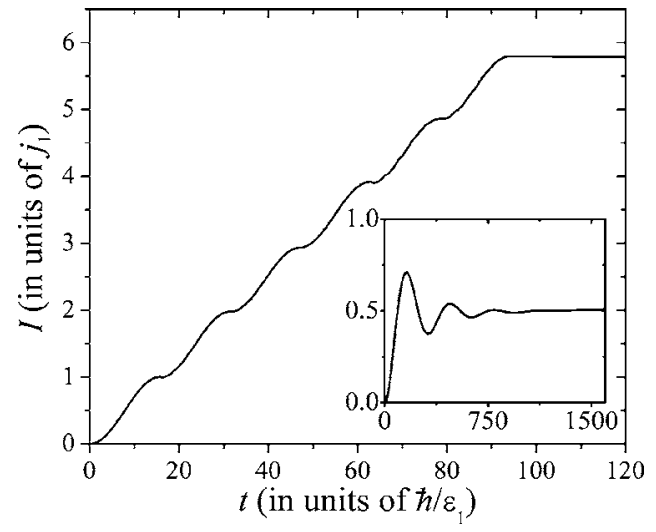

FIG. 4. Current in the ring as a function of time using the proposed pulse sequence. The inset shows for comparison the current in the case of a cw excitation.

oscillations of the diagonal density matrix elements (clearly resolved, for example, for $|0011100\rangle$ at $t \sim 20)$ are due to off-resonant transitions. The current in the ring is calculated as $I=\operatorname{tr}(\rho j)$, where $j$ is the quantum mechanical current operator, and is shown in Fig. 4. Its saturation value at $t$ $\gtrsim 100$ is close to the ideal value $I=6 i_{1}$ predicted by Eq. (7). We show for comparison in the inset of Fig. 4 the current obtained with a cw excitation. The initial oscillation is due to the Rabi oscillation between two states, but the equilibrium value gives a finite value for the current. The Rabi energy in the inset is $\alpha=0.02 \varepsilon_{1}$.

\section{TWO-DIMENSIONAL RINGS}

Experimentally, it is very difficult to fabricate nanoscale narrow quantum rings, whose width $W$ is much smaller than the radius $a$. Typically, self-assembled quantum rings are characterized by $W \simeq a$. Therefore, it is very important to understand the effect of a finite width of the ring on the current generation mechanism by circularly polarized radiation.

Let us consider noninteracting spinless electrons confined into a two-dimensional ring by a radial confinement potential

$$
V(r)=\frac{a_{1}}{r^{2}}+a_{2} r^{2}-V_{0},
$$

where $V_{0}=2 \sqrt{a_{1} a_{2}}$. The potential has a minimum at the average radius $r_{0}=\left(a_{1} / a_{2}\right)^{1 / 4}$ and its effective width at Fermi energy $E_{F}$ is given by $W=\sqrt{8 E_{F} /\left(m^{*} \Omega\right)}$, where $\Omega$ $=\sqrt{8 a_{2} / m^{*}}$.

Without radiation, it can be easily shown in Ref. 18 that the eigenvalues and eigenfunctions of the corresponding Hamiltionian are

$$
\begin{gathered}
E_{m, n}=\left(m+\frac{1}{2}+\frac{M}{2}\right) \hbar \Omega-\frac{m^{*}}{4} \Omega^{2} r_{0}^{2}, \\
m=0,1,2, \ldots, \quad n=\ldots,-1,0,1, \ldots,
\end{gathered}
$$




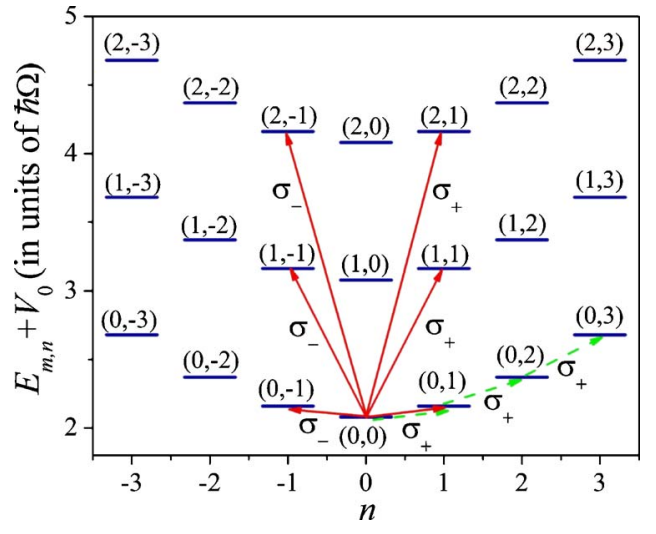

FIG. 5. (Color online) The schematic illustration of the transition scheme in a two-dimensional quantum ring. The straight arrows show possible transition from the ground state $(0,0)$. The dashed arrows represent the transition sequence along the yrast excitation line. This plot was obtained using the parameter value $2 a_{1} m^{*} / \hbar^{2}=10$.

$$
\begin{aligned}
\Psi_{m, n}(r, \varphi)= & R_{m, n}(r) e^{i n \varphi} \\
= & \frac{1}{\lambda}\left(\frac{\Gamma(m+M+1)}{2^{M+1} m ![\Gamma(M+1)]^{2} \pi}\right)^{1 / 2} e^{-r^{2} / 4 \lambda^{2}}\left(\frac{r}{\lambda}\right)^{M} \\
& \times{ }_{1} F_{1}\left[-m, M+1, \frac{r^{2}}{2 \lambda^{2}}\right] e^{i n \varphi},
\end{aligned}
$$

where

$$
\lambda=\sqrt{\frac{\hbar}{m^{*} \Omega}}, \quad M=\sqrt{n^{2}+\frac{2 a_{1} m^{*}}{\hbar^{2}}},
$$

and ${ }_{1} F_{1}$ is the confluent hypergeometric function.

The wave function (10) has the same angular dependence as the wave function (4), thus the matrix elements for dipole transitions $\left\langle m, n|-\mathbf{d E}| m^{\prime}, n^{\prime}\right\rangle$ are nonzero only when $n^{\prime}$ $=n \pm 1$. Generally, the integral over $r$ in the matrix element is nonzero for different $m, m^{\prime}$. Therefore, the circularly polarized radiation can couple energy levels with different $m, m^{\prime}$. Similarly to the derivation given by Eqs. (5) and (6), we can demonstrate that two levels are coupled by $\sigma_{+}(-)$radiation if $E_{m, n+1}-E_{m^{\prime}, n}>(<) 0$ and $\left|E_{m, n+1}-E_{m^{\prime}, n}\right|=\hbar \omega$ (the resonance condition).

Figure 5 demonstrates the transition scheme described above. The straight arrows show the permitted transitions from the ground state $(0,0)$. In contrast to the transition scheme depicted in Fig. 1, the light polarization in the twodimensional ring can control not only the change of the electron angular momentum, but also the radial part of the electron wave function. However, the latter property is not important for the current in the ring. Therefore, we can limit the transitions to yrast line of excitations shown by the dashed arrows in Fig. 5. Notice that the transition along the yrast line can be described by the theory presented in Sec. II with renormalized energy levels and coupling constants. In the limit $2 a_{1} m^{*} / \hbar^{2} \rightarrow \infty$ the energy spectrum (9) coincides with the one-dimensional spectrum (4).

\section{DISCUSSION AND CONCLUSIONS}

The magnetic field along the axis of the ring generated by a current $I$ in the ring can be estimated using $\mathbf{B}$ $=\mathbf{z} \mu_{0} I a^{2} /\left[2\left(z^{2}+a^{2}\right)^{3 / 2}\right]$ where $\mu_{0}$ is the magnetic constant and $\mathbf{z}$ is the unit vector along the axis of the ring. For a GaAs-based quantum rings of $a=10 \mathrm{~nm}\left(m^{*}=0.067 m_{e}\right)$ one finds $i_{1}=0.44 \mu \mathrm{A}$. The magnetic field at the center of the ring, taking into account twofold spin degeneracy and assuming $N_{1}=5$ and $p=5$, gives $B(z=0) \approx 3 \mathrm{mT}$. This value is of the same order of magnitude of the local magnetic field obtained by arrays of nanowires. ${ }^{5}$ The total switching time $T_{s w}$ is given by $T_{s w}=p\left(2 N_{1}+1\right) T_{p}$. Our numerical simulations show that the total current decreases if the Rabi energy becomes comparable to the transition energy. In our simulation we have used $\alpha=0.2 \varepsilon_{1}$ which gives $T_{p} \approx 1.8 \mathrm{ps}$ and $T_{s}$ $\approx 100 \mathrm{ps}$.

The photon spontaneous emission rate is $\gamma_{\xi}^{2} \propto d^{2} \omega_{\xi}^{3}$, with $\hbar \omega_{\xi}=\varepsilon_{m}-\varepsilon_{n}$, and $d$ the dipole moment between the states $|m\rangle$ and $|n\rangle$. Given a typical radius of $10 \mathrm{~nm}$, and $\hbar \omega_{\xi}$ of the order of $5 \mathrm{meV}$, the spontaneous emission rate can be estimated in the order of hundreds of microseconds. It is expected that the main relaxation mechanism is provided by the emission of acoustic phonons through the deformation potential interaction. We have estimated the rate of a single electron relaxation by a single phonon emission and found a characteristic relaxation time of the order of 1 to $10 \mathrm{~ns}$. Notice that the phonon needs to provide angular momentum to the electron system and this decreases the matrix element between the states $|m\rangle$ and $|n\rangle$ with increase of $|n-m|$. This property in combination with the Pauli exclusion principle allows us to conclude that the compact state in Fig. 2(b) is robust against relaxation by phonon emission in the sense that the relaxation time of such a state is much longer than the typical single-electron relaxation time. In the simulation above we have used a conservative value for the relaxation rate of $\gamma=0.025 \varepsilon_{1}$, corresponding to about $500 \mathrm{ps,} \mathrm{which} \mathrm{is}$ shorter than the estimated values for the photon and phonon assisted relaxation times. For larger rings the energy transitions involved in the present scheme will be in the microwave region of the electromagnetic spectrum. Several experiments of quantum control in that spectral range have been reported. ${ }^{19,20}$

In conclusion, we have shown that a strong current can be excited in a quantum ring via a train of phase-locked infrared pulses. The key component in our scheme is the circularly polarization of pulses, which increases the angular momentum of the many-electron state in the ring. We propose to use the system as externally-controlled source of local magnetic fields for single-spin quantum logic.

\section{ACKNOWLEDGMENT}

This research was supported by the National Science Foundation, Grant No. NSF DMR-0312491. 
*Electronic address: pershin@pa.msu.edu

${ }^{1}$ J. R. R. Verlet, V. G. Stavros, R. S. Minns, and H. H. Fielding, Phys. Rev. Lett. 89, 263004 (2002); R. S. Minns, R. Patel, J. R. R. Verlet, and H. H. Fielding, ibid. 91, 243601 (2003).

${ }^{2}$ M. W. Noel and C. R. Stroud, Phys. Rev. Lett. 77, 1913 (1996).

${ }^{3}$ B. E. Kane, Nature (London) 393, 133 (1998); R. Vrijen, E. Yablonovitch, K. Wang, H. W. Jiang, A. Balandin, V. Roychowdhury, T. Mor, and D. DiVincenzo, Phys. Rev. A 62, 012306 (2000); D. Loss and D. P. DiVincenzo, ibid. 57, 120 (1998); G. Burkard, D. Loss, and D. P. DiVincenzo, Phys. Rev. B 59, 2070 (1999).

${ }^{4}$ V. Privman, I. D. Vagner, and G. Kventsel, Phys. Lett. A 239, 141 (1998); Yu. V. Pershin, I. D. Vagner, and P. Wyder, J. Phys.: Condens. Matter 15, 997 (2003).

${ }^{5}$ D. A. Lidar and J. H. Thywissen, J. Appl. Phys. 90, 754 (2004).

${ }^{6}$ A. Lorke, R. J. Luyken, A. O. Govorov, J. P. Kotthaus, J. M. Garcia, and P. M. Petroff, Phys. Rev. Lett. 84, 2223 (2000); J. M. Garcia, G. Medeiros-Ribeiro, K. Schmidt, T. Ngo, J. L. Feng, A. Lorke, J. Kotthaus, and P. M. Petroff, Appl. Phys. Lett. 71, 2014 (1997).

${ }^{7}$ I. D. Vagner, A. S. Rozhavsky, P. Wyder, and A. Yu. Zyuzin, Phys. Rev. Lett. 80, 2417 (1998); V. A. Cherkaskiy, S. N. Shevchenko, A. S. Rozhavsky, and I. D. Vagner, Low Temp. Phys. 25, 541 (1999).

${ }^{8}$ T. Z. Qian, Y. S. Yi, and Z. B. Su, Phys. Rev. B 55, 4065 (1997).
${ }^{9}$ S. N. Shevchenko, Yu. V. Pershin, and I. D. Vagner, Physica E (Amsterdam) 24, 82 (2004).

${ }^{10}$ V. Gudmundsson, C.-S. Tang, and A. Manolescu, Phys. Rev. B 67, 161301(R) (2003).

${ }^{11}$ J. Splettstoesser, M. Governale, and U. Zülicke, Phys. Rev. B 68, 165341 (2003)

${ }^{12}$ B. Molnár, F. M. Peeters, and P. Vasilopoulos, Phys. Rev. B 69, 155335 (2004).

${ }^{13}$ A. Barone, T. Hakioglu, and I. O. Kulik, cond-mat/0203038 (unpublished); I. O. Kulik, J. Phys. B 27, 395 (2003).

${ }^{14}$ T. V. Shahbazyan and S. E. Ulloa, Phys. Rev. B 55, 13702 (1997).

${ }^{15}$ Yu. V. Pershin and C. Piermarocchi (unpublished).

${ }^{16}$ S. Viefers, P. Koskinen, P. Singha Deo, and M. Manninen, Physica E (Amsterdam) 21, 1 (2004).

${ }^{17}$ G. Lindblad, Commun. Math. Phys. 48, 119 (1976).

${ }^{18}$ W.-C. Tan and J. C. Inkson, Semicond. Sci. Technol. 11, 1635 (1996); W.-C. Tan and J. C. Inkson, Phys. Rev. B 53, 6947 (1996); 60, 5626 (1999).

${ }^{19}$ R. G. Mani, J. H. Smet, K. von Klitzing, V. Narayanamurti, W. B. Johnson, and V. Umansky, Nature (London) 420, 646 (2002); Phys. Rev. Lett. 92, 146801 (2004).

${ }^{20}$ M. A. Zudov, R. R. Du, L. N. Pfeiffer, and K. W. West, Phys. Rev. Lett. 90, 046807 (2003). 